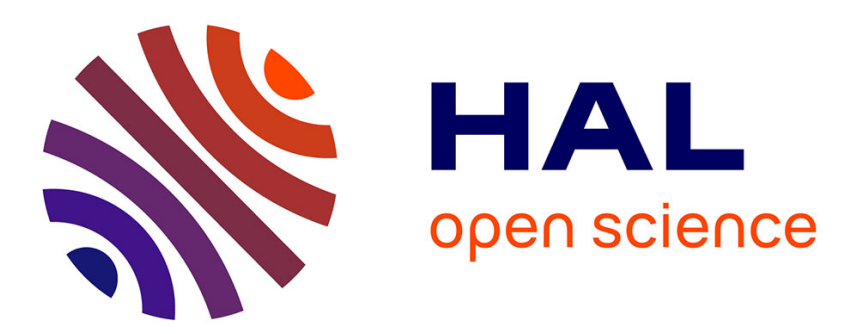

\title{
Mesure des températures locales de surface. Rôle de la microtopographie
}

\author{
Alain-Claude Roudot, M. Chuard, Jean Mignot, D. Rondot
}

\section{To cite this version:}

Alain-Claude Roudot, M. Chuard, Jean Mignot, D. Rondot. Mesure des températures locales de surface. Rôle de la microtopographie. Revue de Physique Appliquée, 1986, 21 (3), pp.245-255. 10.1051/rphysap:01986002103024500 . jpa-00245437

\section{HAL Id: jpa-00245437 https://hal.science/jpa-00245437}

Submitted on 1 Jan 1986

HAL is a multi-disciplinary open access archive for the deposit and dissemination of scientific research documents, whether they are published or not. The documents may come from teaching and research institutions in France or abroad, or from public or private research centers.
L'archive ouverte pluridisciplinaire HAL, est destinée au dépôt et à la diffusion de documents scientifiques de niveau recherche, publiés ou non, émanant des établissements d'enseignement et de recherche français ou étrangers, des laboratoires publics ou privés. 
Classification

Physics Abstracts

44.65

\title{
Mesure des températures locales de surface. Rôle de la microtopographie
}

\author{
A.-C. Roudot (*), M. Chuard (*), J. Mignot et D. Rondot \\ Université de Franche-Comté, I.U.T. de Belfort, rue Engel Gros, 90016 Belfort, France \\ (*) Faculté de Médecine et Pharmacie, Place St-Jacques, 25030 Besançon, France
}

(Reçu le 23 juillet 1984, révisé le 26 novembre 1985, accepté le 4 décembre 1985)

\begin{abstract}
Résumé. - Les auteurs effectuent une étude comparative des caractéristiques géométriques et statistiques du microrelief et de l'image thermique d'une surface. L'analyse de texture de l'image thermique met en évidence une corrélation avec le microrelief et permet de séparer les différentes contributions. La mesure de l'influence du relief et de l'émissivité donne la possibilité d'atteindre une cartographie des températures ponctuelles d'une microsurface. Les phénomènes évolutifs de faible amplitude peuvent être décelés par l'utilisation de l'algorithme de Peucker-Douglas.
\end{abstract}

\begin{abstract}
The authors have done a comparative study between statistical and geometrical characteristics of the microrelief and the thermal image of a surface. The texture analysis of the thermal image shows a correlation with the microrelief and permits the separation of the parameters. Measuring the influence of the relief and the emissivities makes it possible to obtain a cartography of the localized temperatures of a microsurface. The evolution phenomenas of low magnitude can be determined in using Peucker-Douglas' software.
\end{abstract}

\section{Notations.}

$\phi_{i} \quad$ flux thermique mesuré en provenance du point $i$

$\varepsilon_{i} \quad$ émissivité spectrique du point $i$

$T_{i} \quad$ température du point $i$

$\varepsilon_{\mathrm{a}} \quad$ émissivité du milieu ambiant, celui-ci étant considéré comme un milieu homogène, gris, à température constante

$T_{\mathrm{a}} \quad$ température du milieu ambiant

$\alpha \quad$ constante de proportionnalité

$N \quad$ nombre de points de mesure

$\underline{Z}\left(x_{i}\right)$ hauteur du point d'abscisse $x_{i}$

$\bar{Z}$ hauteur moyenne

$L \quad$ longueur totale du profil étudié

$\beta \quad$ distance des deux points entre lesquels la corrélation est établie

$\mu_{i} \quad$ moment centré d'ordre $i$

$f(T)=\frac{\int_{0}^{2} L_{\lambda}(\lambda, T) \cdot \mathrm{d} \lambda-\int_{0}^{\lambda_{1}} L_{\lambda}(\lambda, T) \cdot \mathrm{d} \lambda}{\int_{0}^{\infty} L_{\lambda}(\lambda, T) \cdot \mathrm{d} \lambda} \cdot \frac{\sigma}{\pi} T^{4}$

$\sigma \quad$ constante de Stefan

$L_{\lambda} \quad$ luminance monochromatique d'un corps noir à la longueur d'onde $\lambda$ et à la température $T$ $\lambda_{1}, \lambda_{2}$ limites du spectre $: 8$ et 12 microns.
Remarque. - La caméra travaillant dans le spectre de longueur d'onde 8 à 12 microns, les flux et les luminances spectriques sont définies en intégrant les valeurs monochromatiques sur ce spectre. Les émissivités spectriques sont ensuite définies à partir de ces valeurs intégrées.

\section{Introduction.}

L'image du flux thermique restituée par un détecteur à partir du flux émis par chaque point d'une surface est fonction d'un grand nombre de paramètres. Elle dépend de la température de la surface étudiée mais aussi de la nature et de la forme géométrique du matériau, du microrelief de la surface, de l'angle et de la distance d'observation, des propriétés du milieu ambiant et des caractéristiques du détecteur infrarouge. L'image thermique obtenue n'est pas la représentation des températures réelles de surface. Le microrelief de la surface joue un rôle important dans la formation de l'image thermique. De nombreuses études ont été faites sur les variations du facteur d'émission en fonction des caractéristiques microgéométriques de surface par des méthodes de modélisations bi ou tridimensionnelles [1-6] ou par des mesures comparatives pour évaluer une émissivité apparente dépendant de la rugosité. Les méthodes de calcul se 
heurtent à divers problèmes comme le masquage [7] ou les réflexions multiples $[1,3]$. Des expériences similaires ont aussi été faites sur la réflectivité $[8,9]$. De plus, ces études sont en général limitées à des grandeurs monochromatiques ou totales appliquées à des corps purs. L'utilisation de la thermographie infrarouge pour la mesure des températures de surface implique la connaissance des émissivités dans le domaine de longueur d'onde de fonctionnement de la caméra infrarouge.

\section{Méthode de mesure des températures locales.}

Le flux émis par la surface d'un corps maintenu à température constante n'est pas homogène mais présente des écarts en fonction des variations locales de l'émissivité. Ces variations dépendent du microrelief de la surface. Ainsi quand on considère les flux mesurés entre deux points de la surface d'une pièce maintenue à température constante, on démontre que la variation de flux est proportionnelle à la variation d'émissivité $[10,11]$. En effet, le flux mesuré par la caméra est la somme du flux émis : $\varepsilon_{1} . f\left(T_{1}\right)$ et du flux reçu du milieu ambiant : $\varepsilon_{\mathrm{a}} \cdot f\left(T_{\mathrm{a}}\right)$ et réfléchi vers le détecteur : $\rho_{1} \cdot \varepsilon_{\mathrm{a}} \cdot f\left(T_{\mathrm{a}}\right)$ or $: \rho_{1}+\varepsilon_{1}=1$ d'où :

de même :

$$
\phi_{1}=\varepsilon_{1} \cdot f\left(T_{1}\right)+\left(1-\varepsilon_{1}\right) \cdot \varepsilon_{\mathrm{a}} \cdot f\left(T_{\mathrm{a}}\right)
$$

$$
\phi_{2}=\varepsilon_{2} \cdot f\left(T_{2}\right)+\left(1-\varepsilon_{2}\right) \cdot \varepsilon_{\mathrm{a}} \cdot f\left(T_{\mathrm{a}}\right)
$$

avec : $T_{1}=T_{2}=T$

$$
\Delta \phi=\Delta \varepsilon\left\{f(T)-\varepsilon_{\mathrm{a}} \cdot f(T)\right\}=\alpha . \Delta \varepsilon .
$$

Ces relations valables pour des valeurs monochromatiques et vérifiées pour des valeurs spectriques permettent d'effectuer une étude comparative de la représentation de la distribution des flux superficiels d'un échantillon maintenu à température constante et de la microcartographie de la même zone. Pour l'étude des microsurfaces, le domaine d'application de ces relations a été limité aux faibles valeurs de flux thermiques afin de minimiser les perturbations dues aux réflexions multiples.

L'objet de la présente étude est de mettre au point une méthode d'approche des températures locales, basée sur l'acquisition de deux images :

- L'image infrarouge (en émission ou en réflexion).

- L'image du microreflief obtenue par palpage de la surface.

Ces deux images permettent d'accéder à la cartographie des températures locales. Une telle procédure peut avoir comme application, l'évaluation rapide du rôle du microrelief sur les échanges thermiques en régime permanent ou en régime transitoire entre deux solides en contact (organes de machines) ou entre la paroi d'un solide et un fluide (échangeurs).

Notre étude est effectuée à partir d'un échantillon en aluminium usiné par tournage. Ce procédé d'usi- nage donne naissance à une topographie de surface qui présente un caractère périodique permettant de localiser facilement les accidents du relief.

La surface observée est un carré d'environ $3 \mathrm{~mm}$ de côté. Compte tenu des dimensions de la zone étudiée, de la conductibilité thermique de l'échantillon métallique et des conditions expérimentales, on a considéré que chaque point de la zone observée est à la même température.

Ce mode d'étude peut être appliqué à tous types de surfaces macro ou microrugueuses. Des études ont été réalisées par exemple avec la fonte grise et l'ébonite.

\section{Mesure de la distribution des flux superficiels.}

La chaîne radiométrique de mesure comprend essentiellement une caméra et un système de traitement des informations (Fig. 1). La caméra de thermographie infrarouge utilisée est un pyromètre à balayage opticomécanique qui fournit une image vidéo de la surface étudiée. L'objet est analysé à la fréquence TV par deux miroirs à faible inertie. Au cours de cette analyse, les radiations infrarouges émises par chaque point de l'objet sont focalisées sur un détecteur $\mathrm{HgCd} \mathrm{Te}$ refroidi à l'azote liquide. Le préamplificateur associé à ce détecteur convertit ces radiations infrarouges en une image thermique TV. Le niveau de gris de chaque point de l'image est proportionnel à l'intensité du flux émis par le point considéré. La nature du détecteur $\mathrm{Hg} \mathrm{Cd} \mathrm{Te}$ impose la bande spectrale d'utilisation : 8 à 12 microns. Cette fenetre correspond à une bonne transmission spectrale de l'atmosphère et correspond aux longueurs d'onde du maximum d'émission thermique des corps à la température ambiante.

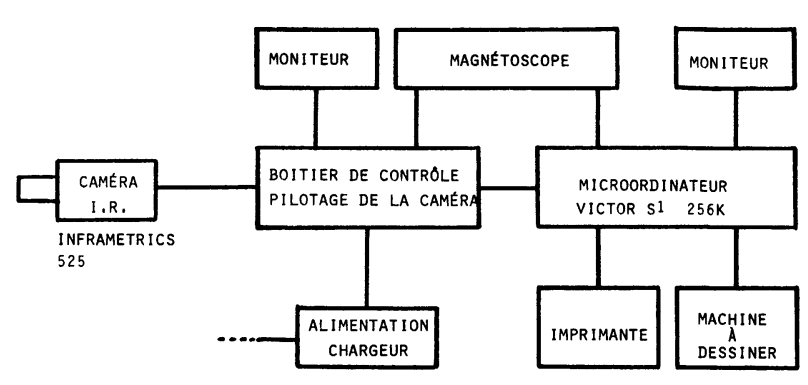

Fig. 1. - Thermographie infrarouge : chaîne de mesure.

[Infrared thermography : measuring device.]

Le système de traitement informatique permet l'analyse fine des informations. Il comprend un microordinateur $(256 \mathrm{~K})$ et ses périphériques. L'image numérisée est stockée sous forme d'une matrice $256 \times 256$ points et 64 niveaux. La représentation tridimensionnelle des flux thermiques provient directement de cette image. Chaque point de l'image représente le flux thermique du point de coordonnées $(x, y)$. La profilométrie thermique est obtenue à partir 
de cette surface par extraction d'une (ou plusieurs) ligne(s) dans une direction déterminée.

Deux méthodes peuvent être utilisées pour établir facilement une microcartographie des flux thermiques qui permettra d'explorer la surface. La première consiste en une mesure par émission, la seconde utilise une mesure par réflexion. Le choix est déterminé par les valeurs des émissivités ou réflectivités de la surface $[12,13]$.

\section{Etude de la microgéométrie de la surface.}

Le montage expérimental comprend un rugosimètre, deux moteurs pas à pas croisés et un microordinateur (Fig. 2). Le rugosimètre travaille avec un palpeur mécanique fixe dans le plan horizontal. Tout déplacement vertical du stylet à induction entraîne une variation de signal électrique (variable entre -5 et $+5 \mathrm{~V}$ ) qui est convertie en signal digitalisé sur 12 bits pour être traité numériquement par le microordinateur. Le palpeur peut travailler avec une référence interne ou sans référence.

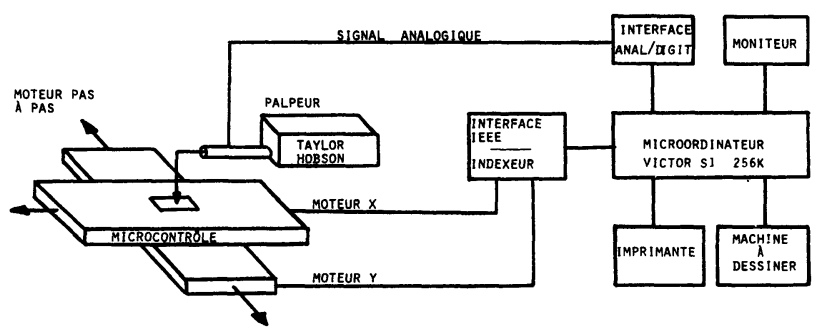

Fig. 2. - Rugosimétrie tridimensionnelle : chaîne de mesure.

[3-D tactile profilometer : measuring device.]

Un profil est obtenu en déplaçant la pièce sous le palpeur dans une direction à l'aide d'un moteur pas à pas. La valeur du déplacement entre deux mesures est programmable et peut être au minimum de 1 micron.

Le passage de l'analyse d'un profil à celui d'une surface est réalisé en décrivant une succession de lignes parallèles équidistantes grâce au deuxième moteur pas à pas. La connaissance de la cote $z$ du palpeur associée à celle des coordonnées $x$ et $y$ repérant les positions de chacun des deux moteurs croisés permet de connaître la surface suivant une loi : $z=$ fonction $(x, y)$ connue en chaque point.

Le schéma de principe du montage expérimental montre les principaux éléments de la chaîne :

- le palpeur

- les moteurs pas à pas

- l'indexeur de commande des moteurs pas à pas

- le microordinateur (256 K)

- l'imprimante avec copie d'écran

- la machine à dessiner.
L'image digitalisée est stockée sous la forme d'un fichier $256 \times 256$ points et 4096 niveaux (12 bits) ramenés à 256 niveaux ( 8 bits) pour l'étude des surfaces ou d'un nombre maximum de 4000 points pour celle des profils.

\section{Méthodes d'analyse.}

La mesure des températures locales de surface nécessite la comparaison des images obtenues par rugosimétrie bi ou tridimensionnelle et par thermographie infrarouge.

5.1 ProfilométrIE GÉOMÉTRIQUE ET PROFILOMÉTRIE THERMIQUE. - Le profil géométrique du relief peut être obtenu de deux manières différentes :

- par enregistrement direct avec le palpeur dans une direction choisie de l'échantillon;

- par reconstitution d'une ligne à partir de l'image d'une surface.

Le profil thermique est extrait de l'image thermique de la surface.

Le profil géométrique ou thermique ainsi constitué (4 000 points maximum) est stocké en mémoire de masse et de nombreuses études peuvent être effectuées après élimination du défaut de forme ou de l'ondulation par une méthode de moyenne glissante [14]. Ces opérations correspondent à un filtrage passe-haut (ou passe-bas) qui permet la séparation des différentes composantes significatives d'un profil réel.

L'analyse statistique du profil corrigé peut alors être effectuée. La terminologie de la rugosimétrie est utilisée pour la définition des critères statistiques des profils thermiques [15-17] :

- La rugosité moyenne :

$R_{\mathrm{a}}=\frac{1}{N} \sum_{i=1}^{N}\left|Z\left(x_{i}\right)\right|$ avec $\left|Z\left(x_{i}\right)\right|=\left|Z\left(x_{i}\right)-\bar{Z}\right|$.

- La rugosité maximum : $Z_{\max }-Z_{\min }$.

- L'écart-type $\sigma$ (ou la variance $\sigma^{2}$ ) de la fonction de distribution des hauteurs :

$$
\mu_{2}=\sigma^{2}=\int_{-\infty}^{+\infty}(Z-\bar{Z})^{2} \cdot P(Z) \cdot d Z
$$

où $P(Z)$ est la densité de distribution des hauteurs.

- Le paramètre d'obliquité ou Skewness $S_{\mathbf{K}}$ :

$S_{\mathrm{K}}=\frac{\mu_{3}}{\left(\mu_{2}\right)^{3 / 2}}=\frac{\mu_{3}}{\sigma^{3}}$ avec $\mu_{i}=\int_{-\infty}^{+\infty}(Z-\bar{Z}) \cdot P(Z) \cdot \mathrm{d} Z$.

- Le paramètre d'aplatissement ou Kurtosis :

$$
E_{\mathrm{K}}=\frac{\mu_{4}}{\left(\mu_{2}\right)^{4 / 2}}=\frac{\mu_{4}}{\sigma^{4}}
$$

Ces deux paramètres sont intéressants lors de la comparaison de la fonction de distribution des hau- 
teurs avec une distribution gaussienne ou lors de l'étude d'un phénomène évolutif.

- L'étalement horizontal du profil est étudié par la fonction d'autocorrélation :

$$
R_{(\beta)}=\frac{1}{\sigma^{2}(L-\beta)} \int_{0}^{L-\beta} Z(x) \cdot Z(x+\beta) \cdot d x .
$$

Cette fonction décèle les éventuelles périodicités dans la distribution des aspérités.

- A partir de la fonction de densité de probabilité $P(z)$, deux fonctions sont accessibles :

- La fonction de répartition des hauteurs qui représente le pourcentage des hauteurs des aspérités comprises entre $z$ et $z+\mathrm{d} z$.

- La fonction de répartition des hauteurs (courbe d'Abbott) donnant les fréquences cumulées.

- L'analyse de Fourier des profils :

L'algorithme employé pour effectuer la transformation de Fourier et son inverse est celui de CooleyTuckey [18] qui utilise un procédé de dichotomie consistant à ramener le traitement de $N$ points au traitement de deux fonctions de $N / 2$ points.

L'étude des différentes fréquences d'un signal est intéressante, elle permet de vérifier l'isotropie d'une surface et de mettre en évidence les composantes périodiques prépondérantes.

5.2 Cartographie DES FluX ET TOPOGRAPHIE DE SURFACE. - Avant tout traitement des images digitalisées $(256 \times 256$ points, 64 ou 256 niveaux $)$, il est possible, si nécessaire, de supprimer le défaut de forme par une méthode des moindres carrés (suppression d'un défaut plan) ou d'effectuer un lissage (par une méthode de moyenne glissante analogue à celle utilisée pour les profils) dont le but est de séparer les contributions caractéristiques. Le fichier ainsi constitué permet pour l'image thermique et pour l'image du microrelief :

- Le tracé :

- de l'image tridimensionnelle

- des courbes de niveau

- des coupes à différents niveaux

- du gradient de l'image (dérivée première du " relief » de l'image) l'image par rapport aux coordonnées $x, y$ et $z$, définie en chaque point par :

$$
z^{\prime}=\sqrt{1+\left(\frac{\partial z}{\partial x}\right)^{2}+\left(\frac{\partial z}{\partial y}\right)^{2}}
$$

- de l'histogramme de distribution des hauteurs

- de l'histogramme des hauteurs cumulées ou courbe de portance.

- Le calcul des paramètres de la distribution des hauteurs de l'image (écart type $\sigma$, paramètres $S_{\mathrm{K}}$ et $E_{\mathrm{K}}$ ).

- La localisation et la distribution de points particuliers tels que sommets, creux, vallées, crètes, cols à partir de l'algorithme de Peucker-Douglas [17].
-- La correction de l'image des flux thermiques par l'image caractéristique de la distribution des émissivités.

Contrairement aux études classiques qui utilisent les données issues de l'analyse de profils (2 dimensions), les critères statistiques ou paramètres caractéristiques déduits de l'étude d'une image tridimensionnelle sont représentatifs des propriétés de cette image car les résultats ne sont plus, dans ce cas, dépendants de l'anisotropie. L'exemple utilisé dans notre étude (échantillon fortement anisotrope) démontre bien la nécessité d'opérer sur des relevés tridimensionnels.

L'étude comparative de l'image thermique et de l'image du microrelief n'est possible que grâce au calage rigoureux d'une image par rapport à l'autre en utilisant des repères caractéristiques indépendants de la méthode de mesure. Cette procédure permet de corriger en même temps, les déformations relatives éventuelles de l'une des images par un système de détection.

Les transformations permettant la superposition des images issues des deux systèmes d'acquisition consistent en une dilatation ou contraction dans une ou deux directions, un effet zoom avec ou sans changement d'origine, une rotation d'angle quelconque et de centre quelconque ou une combinaison de ces transformations.

\section{Résultats expérimentaux.}

L'image représentative des émissivités locales de la surface va permettre, à partir de la distribution des flux thermiques, d'obtenir la cartographie des températures locales. La procédure expérimentale comporte deux étapes successives :

- L'analyse comparative de la topographie de surface et de la cartographie des émissivités.

- L'étude de l'évolution des températures locales de surface en fonction de la microtopographie.

6. 1 MiCROTOPOGRAPHIE ET ÉMISSIVITÉs. - Dans cette première étape, l'acquisition des données par thermographie infrarouge est effectuée à température constante $\left(22^{\circ} \mathrm{C}\right)$. Cette température n'est pas mesurée avec une grande précision, il est cependant nécessaire pour le calibrage de la caméra infrarouge que cette température appartienne à l'échelle de température utilisée $\left(20\right.$ à $\left.50^{\circ} \mathrm{C}\right)$. La distribution des flux représente alors la répartition des émissivités pour cette gamme de températures.

Le rôle de la topographie sur l'émissivité de la surface ne peut être étudié qu'à partir des deux fichiers de la même zone obtenus après traitement de mise en coïncidence et après lissage du microrelief. En effet, le pas de mesure en topographie étant de 13 microns, la résolution spatiale en thermographie de l'ordre de 90 microns, un lissage du fichier de la microsurface est donc nécessaire pour obtenir une définition comparable dans les deux images. Ce lissage est 
effectué par moyenne glissante en centrant un carré de $7 \times 7$ points sur le point considéré et en attribuant la moyenne arithmétique des valeurs de ces 49 points au point central. Ce calcul est opéré en chaque point de l'image en tenant compte des frontières.

\subsubsection{Analyse qualitative.}

- Représentations tridimensionnelles.. - La texture générale de l'échantillon est confirmée à la fois par l'image du microrelief (Fig. 3) et par celle de la distribution des flux thermiques (Fig. 4). On retrouve sur l'image thermique les périodicités laissées par l'usinage. Cependant les réflexions multiples qui ont lieu dans les creux des sillons renforcent le flux thermique issu de ces zones et accroissent la disparité entre les deux images.
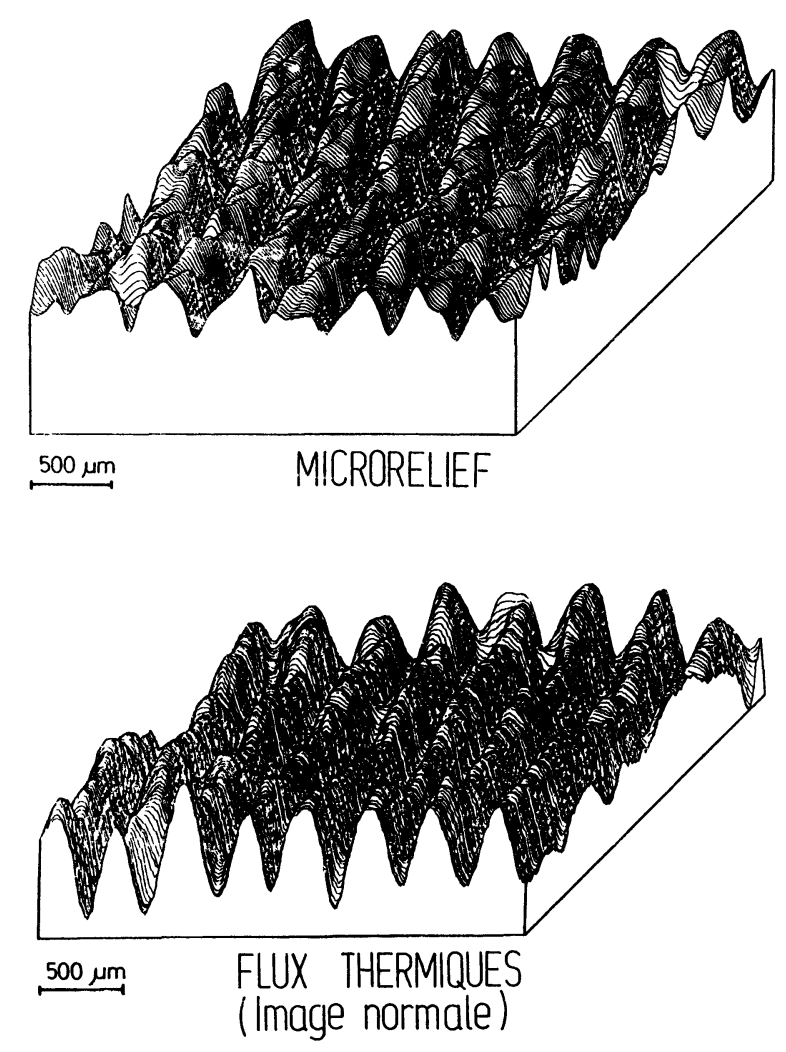

Fig. 3. - Microrelief et flux thermique.

[Microrelief and thermal flux.]

- Courbes de niveau et coupes à différents niveaux. - Deux points de calage (empreintes pyramidales d'essai de dureté Vickers repérées Fig. 4 par $X$ et $Y$ ) sont utilisées pour la mise en coïncidence des images. Les réflexions multiples perturbent la représentation de ces repères dans l'image infrarouge. L'étude des images du relief géométrique et thermique permet de mettre en évidence, dans chaque cas, les perturbations dues aux arrachements du métal lors de l'usinage de l'échantillon.
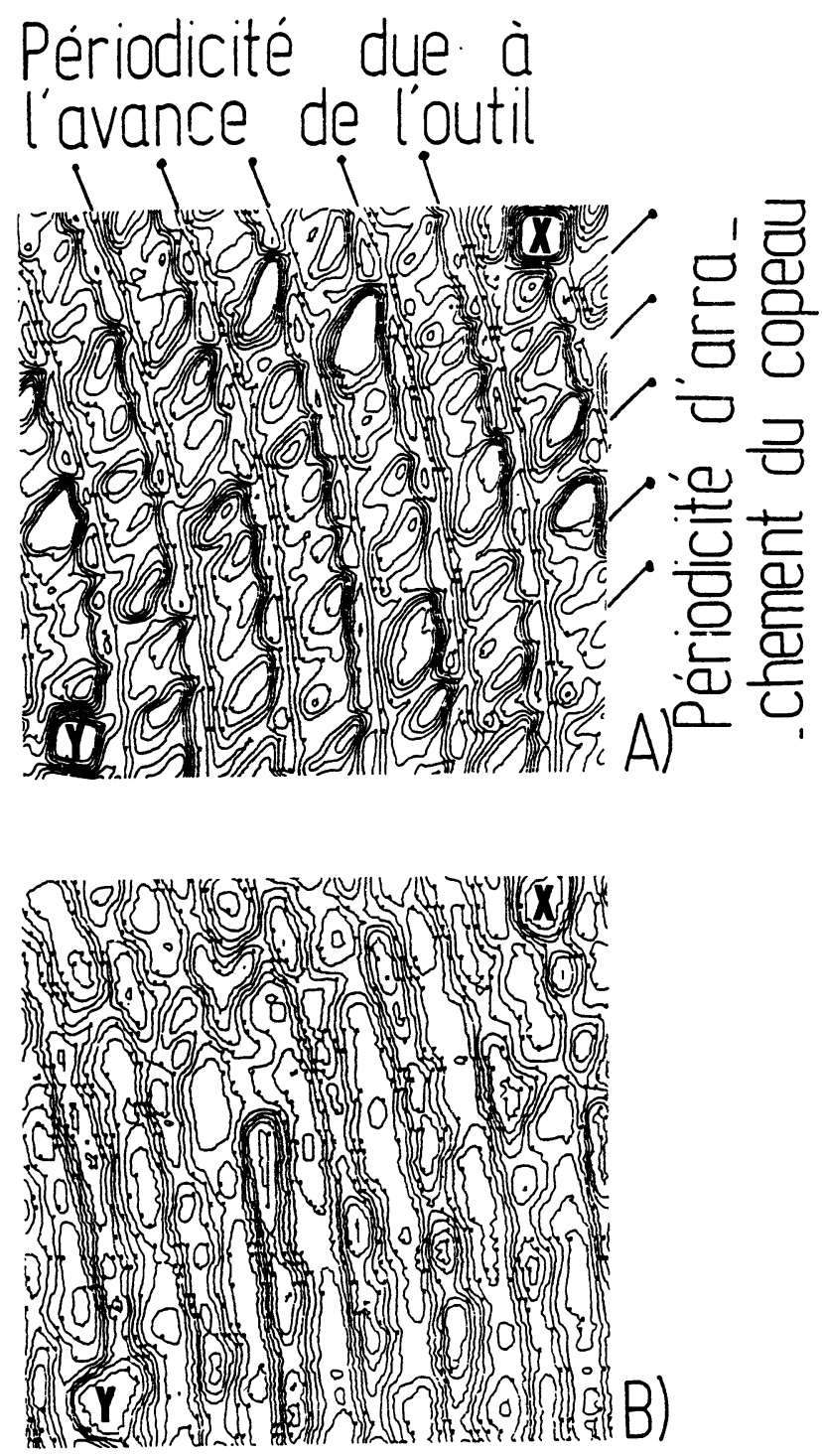

$750 \mu \mathrm{m}$

Fig. 4. - Courbes de niveaux. A : microrelief; B : image thermique.

[Contours; A : microrelief; B : thermal image.]

- En creux de sillon, les variations de flux de l'image infrarouge sont accentuées par les réflexions multiples. La largeur de la vallée est renforcée à la fois par ce phénomène et par la forme de l'indicatrice des émissivités [3].

- En sommet de sillon, le microrelief met en évidence les périodicités d'arrachement du copeau. L'image infrarouge atténue les phénomènes de faible amplitude et renforce les zones d'arrachement qui sont séparées par une perturbation conséquente du relief (Fig. 4).

La morphologie générale de l'image est identique dans les deux cas. Cette constatation est confirmée par les coupes effectuées à différents niveaux. 
- Squelettisation à partir de l'algorithme de Peucker-Douglas. - L'algorithme de Peucker-Douglas [19] permet de détecter, compter, classer ou représenter les éléments constitutifs de la surface tels que : sommets, cols, vallées, crêtes, ravins... (Fig. 5). La définition de chacun de ces points correspond à celle utilisée en cartographie.
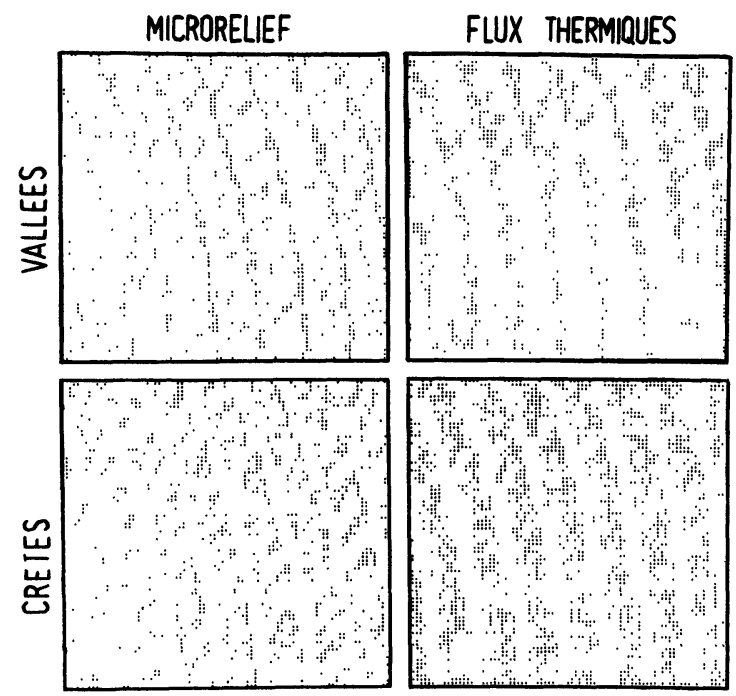

Fig. 5. - Localisation des vallées et des crêtes.

[Crests and valleys localization.]

Cette représentation permet, par superposition à la surface d'origine, de localiser les perturbations caractéristiques du relief et celles de l'image thermique.

La détection des vallées situe toujours nettement la direction des sillons et celle des arrachements de forte amplitude mais ne met pas en évidence sur l'image thermique toutes les périodicités du relief.

Ainsi cette méthode d'analyse d'une famille de points particuliers de la surface permettra de déceler des phénomènes thermiques de faible amplitude que l'étude globale de l'ensemble des points ne peut mettre en évidence.

6.1.2 Analyse quantitative. - L'étude statistique permet de quantifier les analogies entre les deux images et de sélectionner les paramètres qui donnent des résultats similaires et ceux qui traduisent la spécificité du relief thermique.

Afin de mieux comparer les résultats, la corrélation effectuée avec le microrelief d'origine (non lissé). Les paramètres suivants ont été étudiés :

- Hauteurs, pentes, courbures. - Les distributions des hauteurs (Fig. 6) sont très proches entre les images du microrelief d'origine et du microrelief lissé. Le lissage provoque l'atténuation des hautes fréquences. Ceci est vérifié par la valeur du paramètre d'aplatissement ou Kurtosis de la surface lissée qui est supérieure à celle de la surface d'origine.
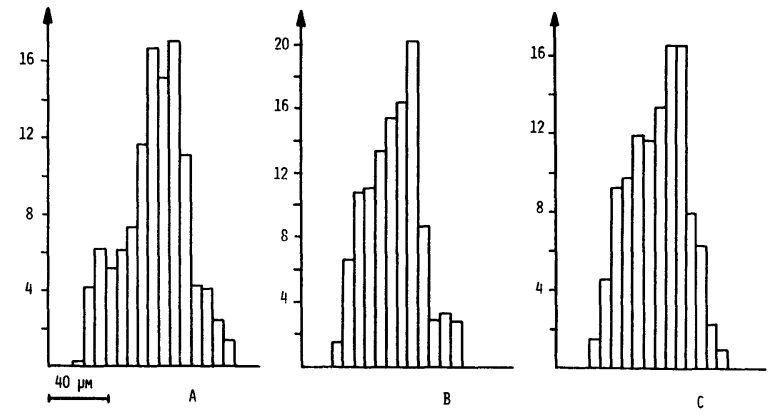

Fig. 6. - Distribution des hauteurs. A : microrelief; B : microrelief lissé; $\mathrm{C}$ : image thermique.

[Height distribution. A : microrelief; B : microrelief after smoothing; $\mathrm{C}$ : thermal image.]

Les résultats de l'étude statistique sur les pentes et les courbures regroupés sous la forme d'un tableau comparatif (Fig. 7), mettent en évidence le rôle du lissage et sa nécessité afin de procéder à l'étude sur des images ayant la même définition. Les valeurs obtenues à partir de la surface lissée sont plus voisines de celles du relief thermique que celles qui proviennent de la surface d'origine. La variance et l'écart-type donnent dans chaque cas, des résultats identiques.

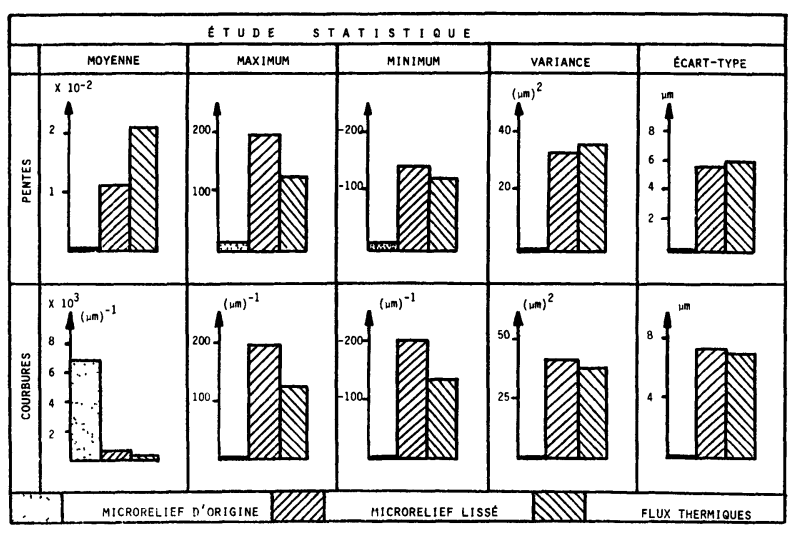

Fig. 7. - Etude statistique sur les pentes et les courbures.

[Statistical study on slopes and curvatures.]

- Analyse de Fourier (Fig. 8). - L'analyse de Fourier du microrelief lissé et de la distribution des flux thermiques met en évidence la similitude des distributions spatiales et les mêmes fréquences principales. Ces fréquences se retrouvent parmi d'autres fréquences caractéristiques dans l'analyse spectrale du microrelief d'origine.

- Autocorrélation (Fig. 9). - L'analyse spectrale des fonctions d'autocorrélation du microrelief lissé et de la distribution des flux confirme l'existence des mêmes composantes périodiques prépondérantes. $\mathrm{La}$ fonction d'autocorrélation est dans chaque cas consti- 

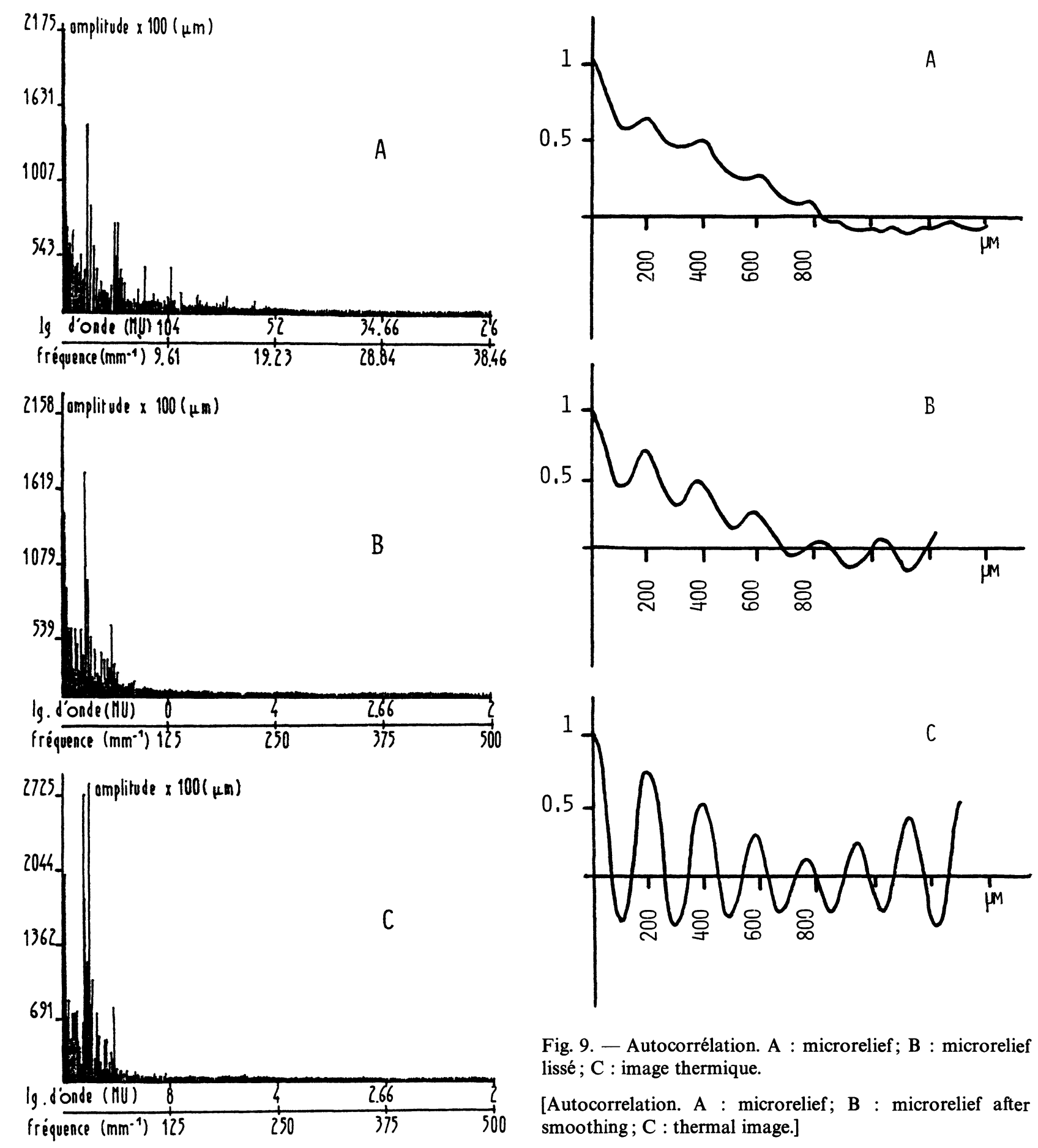

Fig. 9. - Autocorrélation. A : microrelief; B : microrelief lissé; $\mathrm{C}$ : image thermique.

[Autocorrelation. A : microrelief; B : microrelief after smoothing; $\mathrm{C}$ : thermal image.]

Fig. 8. - Analyse de Fourier. A : microrelief; B : microrelief lissé; $\mathrm{C}$ : image thermique.

[Fourier analysis. A : microrelief; B : microrelief after smoothing; $\mathrm{C}$ : thermal image.]

tuée par deux signaux périodiques modulés l'un par l'autre, l'amplitude et la périodicité du signal de modulation se retrouvent dans cette fonction.

6.2 Températures locales De SURFACE. - La cartographie des températures locales de surface à un instant donné permet à la fois l'étude comparée des

températures avec les flux thermiques ou le relief et le suivi des phénomènes thermiques évolutifs.

6.2.1 Mesure des températures. - La représentation des températures locales de surface est obtenue à partir de la distribution des flux thermiques et de la cartographie des émissivités. La méthode de correction consiste à enregistrer d'abord l'image de la surface à température constante. Le résultat caractérise les émissivités. Chaque point de la distribution des flux thermiques de la même surface soumise à un phénomène d'échange est ensuite divisé par un coefficient proportionnel à la valeur de l'émissivité locale 
extraite de l'image précédente. Cette correction permet d'obtenir la cartographie des températures réelles de surface par calibrage des différents niveaux de températures.

L'étude comparée montre que les histogrammes des hauteurs, pentes et courbures de l'image des flux thermiques présentent une distribution plus étalée que ceux des températures. L'écart réel de température est faible en comparaison du contraste de l'image des flux thermiques (Fig. 10).

6.2.2 Phénomène évolutif. - Une des applications importarites de la méthode proposée est l'étude des températures de surfaces lors d'un phénomène évolutif de faible amplitude.

Une surface rugueuse portée à une certaine température est soumise à un refroidissement forcé.

L'évolution thermique de la surface est suivie par caméra de thermographie infrarouge. Un phénomène transitoire de courte durée apparaît en début d'expérience avant l'établissement d'un équilibre dans les échanges thermiques. Un ensemble d'images réparties sur deux secondes va permettre d'établir l'importance des points particuliers du microrelief lors de l'étude
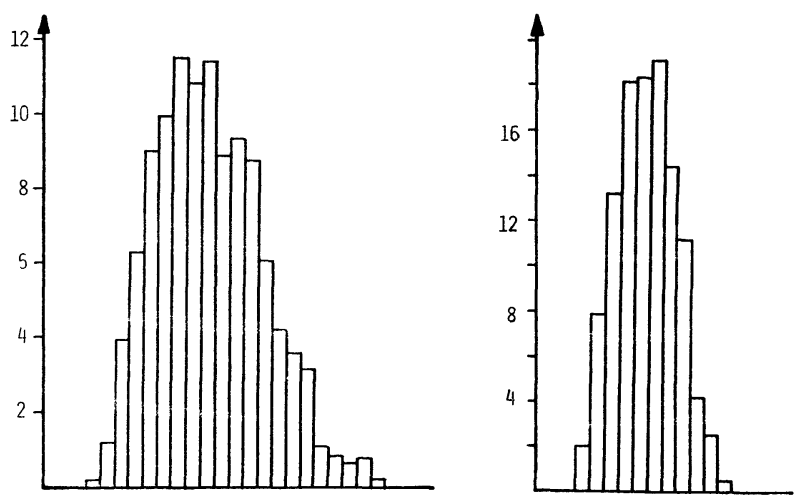

50 DIGITS

B

Fig. 10. - Distribution des flux thermiques (A) et distribution des températures (B).

[Thermal flux distribution (A) and temperature distribution (B).]

de la phase initiale du refroidissement. L'évolution du phénomène est suivie par analyse statistique sur les images des distributions des flux thermiques et sur
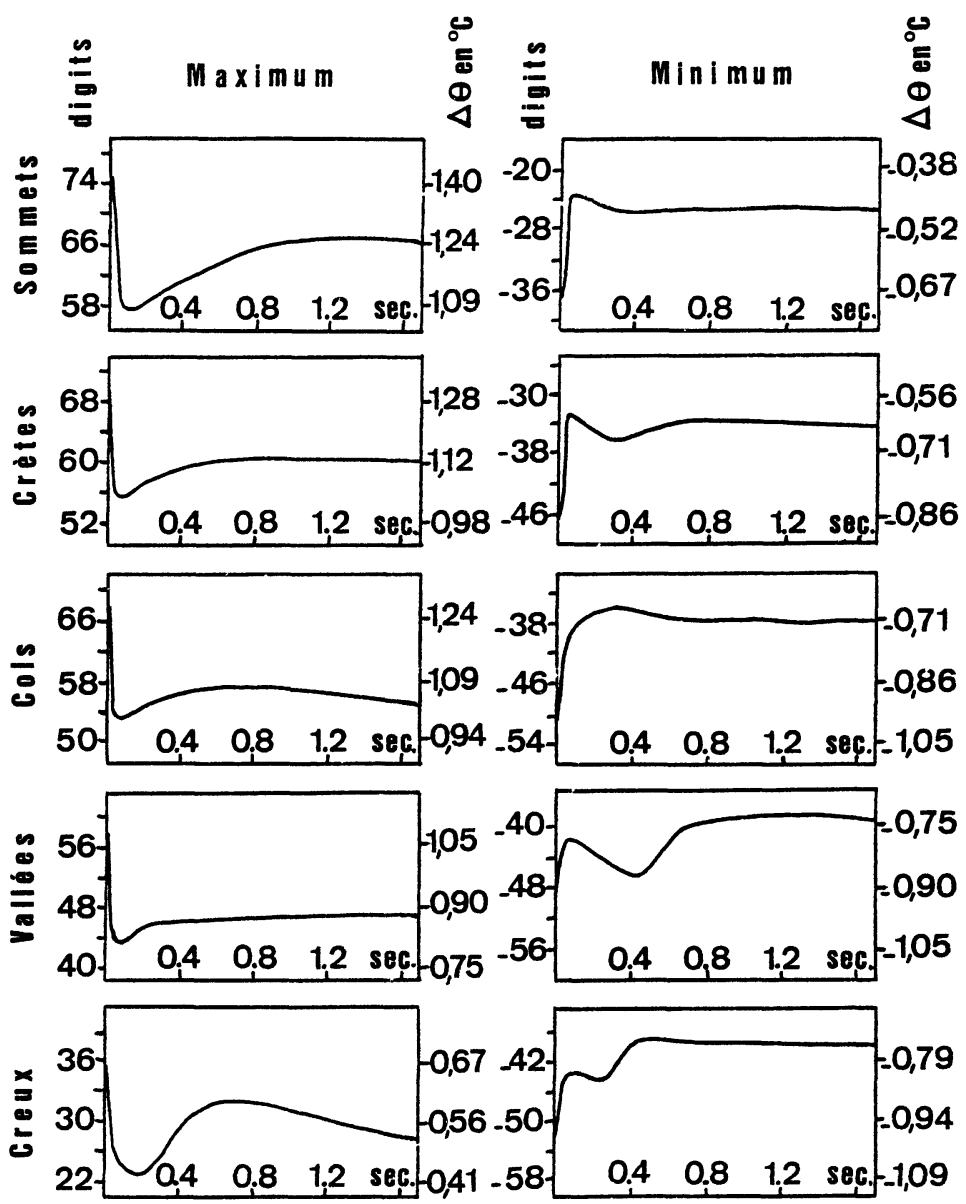

Fig. 11. - Evolution des températures (maximum et minimum) des points particuliers.

[Temperature evolution (maximum and minimum) of particular points.] 
les représentations des températures locales. L'analyse statistique sur 4000 points extraits de l'image des températures ne montre pas l'existence du phénomène transitoire de faible amplitude. La prise en compte de l'ensemble des points produit un effet de moyenne sur les résultats. Seule l'étude des points particuliers permet de déceler et suivre les variations de température. L'évolution en fonction du temps des positions des maxima et minima de la distribution statistique des sommets, crêtes, cols, vallées et creux de températures fait apparaître le rôle spécifique des points particuliers sur les températures en début d'expérience (Fig. 11). Les écarts de chaque courbe sont faibles : à 50 digits correspond un écart de température de $0,94^{\circ} \mathrm{C}$. Le calibrage des écarts de température est effectué pour chaque type de relief. Néanmoins, il apparaît des similitudes dans les formes de courbes de variation de température des maxima et minima de certaines familles : sommets et crêtes ou creux et vallées. Les microreliefs semblables entraînent les mêmes types de variations de températures. Seules, les variations de températures ont été mesurées pour éviter en partie notamment au niveau des vallées et des creux l'erreur introduite par les réflexions multiples sur la valeur absolue de la température de chaque point.

\section{Conclusions.}

Ce travail a permis de contribuer à l'interprétation $\mathrm{du}$ thermogramme d'une surface microrugueuse. L'image thermique d'une microsurface est toujours très dépendante du relief et la mesure des variations de température locale à partir de la distribution des flux thermiques impose l'élimination des effets de la topographie. La thermographie infrarouge donne la possibilité malgré sa spécificité d'appréhender avec une très grande rapidité de la prise de données et avec une bonne précision, certaines caractéristiques géométriques ou statistiques d'un microrelief. L'utilisation du couplage : microrugosimétrie tridimensionnelle tactile et thermographie infrarouge permet la corrélation des variations de températures de surface avec le relief. La cartographie des émissivités peut, en effet être établie à partir du relief numérique et de l'indicatrice des émissivités directionnelles du matériau examiné ou à partir d'un relevé des flux à température constante. Chaque point de l'image tridimensionnelle de la distribution des flux étant formé essentiellement dans certaines conditions par la contribution de l'émissivité et de la température, ces deux effets peuvent être séparés. A partir de la cartographie des températures, deux analyses statistiques peuvent être développées :

- L'une sur un relevé profilométrique de 4000 points successifs.

- L'autre sur les points caractéristiques de l'image.

La première méthode permet l'étude des phénomènes thermiques présentant des variations sensibles de températures. La seconde est plus adaptée aux faibles variations liées à la microgéométrie de la surface. On montre qu'un phénomène transitoire rapide qui n'a pas été décelé par une analyse statistique classique sur les hauteurs, pentes et courbures de la distribution des températures a pu être mis en évidence par une analyse statistique portant uniquement sur les points particuliers. Cette possibilité sera utile dans les études de phénomènes d'échanges thermiques en fonction de l'état géométrique ou de la porosité de la surface. 
Annexe.

Procédure utilisée pour la comparaison de la topographie et de la distribution des flux thermiques d'une même surface.

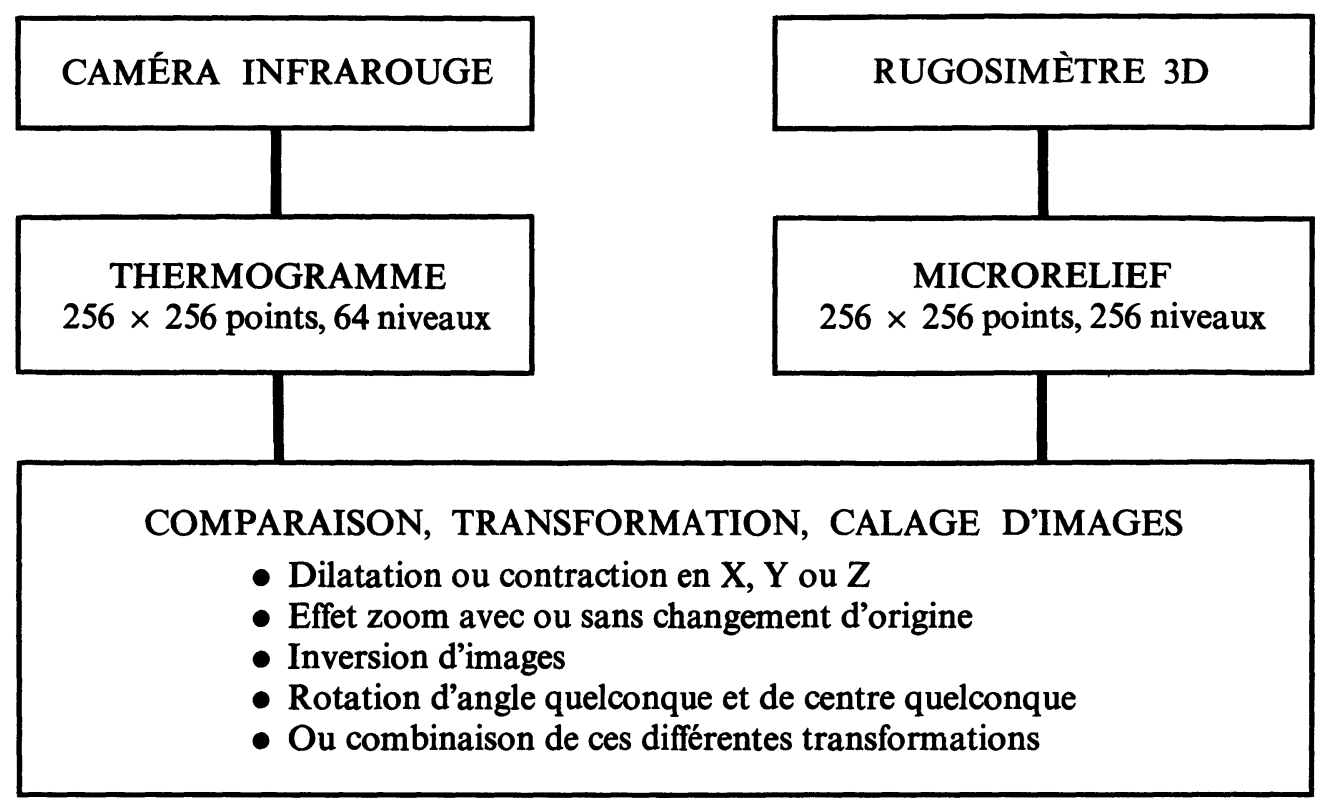

\begin{tabular}{|c|c|}
\hline \multicolumn{2}{|c|}{ TRAITEMENT ET ANALYSE } \\
\hline PROFILOMÉTRIE & REPRÉSENTATION 3D \\
\hline $\begin{array}{l}\text { CONSTITUTION D’UN PROFIL : } \\
\text { - Relevé direct } \\
\text { - Extraction d'un profil d'une image } 256 \times 256 \\
\text { - Points dans une direction quelconque } \\
\text { - Juxtaposition de profils }\end{array}$ & $\begin{array}{l}\text { CONSTITUTION D’UNE REPRÉSENTATION } \\
\text { TRIDIMENSIONNELLE }\end{array}$ \\
\hline $\begin{array}{l}\text { LISSAGE D'UN PROFIL } \\
\text { - Défaut de forme } \\
\text { - Rugosité } \\
\text { - Ondulation }\end{array}$ & $\begin{array}{l}\text { LISSAGE D'UNE SURFACE } \\
\text { - Défaut de forme } \\
\text { - Rugosité } \\
\text { - Ondulation }\end{array}$ \\
\hline $\begin{array}{l}\text { ANALYSE STATISTIQUE SUR LES HAUTEURS, } \\
\text { PENTES ET COURBURES } \\
\text { - Valeur moyenne } \\
\text { - Valeur maximum } \\
\text { - Valeur minimum } \\
\text { - Ecart-type } \\
\text { - Variance } \\
\text { - Paramètre d'obliquité ou skewness } \\
\text { - Paramètre d'aplatissement ou kurtosis } \\
\text { - Autocorrélation } \\
\text { - Densité de distribution } \\
\text { - Fréquences cumulées } \\
\text { - Transformée de Fourier }\end{array}$ & $\begin{array}{l}\text { REPRÉSENTATION TRIDIMENSIONNELLE } \\
\text { - Addition, soustraction d'images } \\
\text { - Multiplication par un coefficient réel } \\
\text { - Courbes de niveaux } \\
\text { - Coupes à différents niveaux } \\
\text { - Gradient de l'image } \\
\text { - Histogramme des distributions des hauteurs } \\
\text { - Courbe de portance } \\
\text { - Surface développée } \\
\text { - Ecart-type } \\
\text { - Skewness } \\
\text { - Kurtosis } \\
\text { - Correction d'une surface à partir d'une autre et } \\
\text { d'un fichier de correction } \\
\text { - Localisation des points particuliers (sommets, } \\
\text { cols, vallées, creux), histogrammes, statistiques }\end{array}$ \\
\hline
\end{tabular}




\section{Bibliographie}

[1] Sacadura, J. F., Modélisation et étude expérimentale du rayonnement thermique de surfaces métalliques microrugueuses. Thèse ès Sciences - Lyon (1980).

[2] Kanayama, K. et Baba, H., Directional monochromatic emittance of the random rough surfaces of metals and non metals. Heat Transfer. Japan. Res. 5 (1976) 1-30.

[3] HERvÉ, P., Influence de l'état de surface sur le rayonnement thermique des matériaux solides. Thèse ès Sciences - Paris (1977).

[4] DRUElle, P., Influence de macrorugosités sur les caractéristiques radiatives de certaines surfaces. Applications aux aciers inoxydables. Thèse DocteurIngénieur - Paris (1978).

[5] Tran n'Guyen, H., Calcul des propriétés radiatives des surface macrorugueuses par la méthode de Monte Carlo. Thèse Docteur-Ingénieur - Paris (1981).

[6] Demont, P., Caractéristiques radiatives des aciers ionoxydables dans le domaine du visible. Schématisation de la rugosité à l'aide de calottes sphériques et étude d'un système solaire hémisphérique. Thèse Docteur-Ingénieur - Paris (1980).

[7] BirkebaK, R. C. et Abdulkadir, A., Random rough surface model for spectral directional emittance of rough metal surface. Int. J. Heat Mass Transfer 2 (1976) 1039-1043.

[8] BirkebaK, R. C. et ECKERT, E. G., Effects of roughness of metal surfaces on angular distribution of monochromatic reflected radiation. J. Heat Transfer 2 (1965) 85-94.

[9] Torrance, K. E. et Sparrow, E. M., Biangular reflectance of an electric non conductor as a fonction of wavelength and surface roughness. J. Heat Transfer 5 (1965) p. 283-292.

[10] Martin, C., Contribution à l'utilisation de la thermo- graphie infrarouge pour les mesures de flux de température et de divers paramètres. Thèses ès Sciences. Limoges (1978).

[11] Martin, C. et Fauchais, P., Mesure par thermographie infrarouge de l'émissivité de matériaux bons conducteurs de la chaleur. Influence de l'état de surface, de l'oxydation et de la température. Revue Phys. Appl. 15 (1980) 1469-1478.

[12] Huetz-Aubert, M. et Sacadura, J. F., Mesure des émissivités et des réflectivités monochromatiques directionnelles des matériaux opaques. Revue Phys. Appl. 17 (1982) 251-260.

[13] Demont, Ph., Tran Nguyen, H. et Sacadura, J. F., Influence de l'oxydation et de la rugosité sur les caractéristiques radiatives des aciers inoxydables. J. Physique. Coll. (1981) 42 (1981) C1-161-172.

[14] ChuARD, M., Rondot, D. and Mignot, J., Numerical filtering used in microtopographical surfaces measurement. WEAR 97, 3 (1984) 267-274.

[15] Thomas, T. R., Recent advances in the measurement and analysis of surface microgeometry. WEAR 33 (1975) 205-233.

[16] Gorecki, C., Tribillon, G., Mignot, J., Profilométrie optique en lumière blanche. J. Optics 14-1 (1983) 19-23.

[17] Chuard, M., Roudot, A.-C., Mignot, J., On the use of a modular system for microtopographical surfaces measurement, WEAR 96, 1 (1984) 31-44.

[18] Cooley, J. W., TuCKeY, J. W., An algorithm for the machine calculation of complex Fourier series. Math. Comput 19 (1965) 297-301.

[19] Peucker, T. K., Douglas, D. H., Detection of surface specific points by local processing of discrete terrain evaluation data. Computer Graph. Im. Processing 4 (1975) 375-387. 